\title{
The Real Intraoperatve Diagnosis of a Patient with Lipothymia and Arterial Hypotension
}

\section{Manuela Stoicescu*}

Faculty of Medicine and Pharmacy, Medical Disciplines Department, University of Oradea, Romania

\begin{abstract}
Objectives: The main objectives of this clinical case presentation was to determine the real cause of a 50 year patient that presented into emergency service for an episode of lipothymia and arterial hypotension (BP=60/40 $\mathrm{mmHg}$ ).

Description of the methods: I am presenting the clinical case of a 50 year old patient that come at the emergency service for an episode of lipothymia and arterial hypotension $(B P=60 / 40 \mathrm{mmHg})$. The objective examination revealed: pale skin and mucous, tachycardia $(H R=105 / \mathrm{min})$, normal vesicular sound, $B P=60 / 40 \mathrm{mmHg}$. Palpation of the abdomen was insensitive, soft, elastic, participating at the respiratory movements. No presented rebound tenderness (without clinical signs of peritoneal irritation). Normal rectal touch-brown aspect of feces without pain during the maneuver. The paraclinical investigations have shown: EKG sinus tahycardia (105 bates/minutes), ESR=24/42, fibrinogen=580 mg/dl, $\mathrm{Hb}=11 \mathrm{~g} / \mathrm{dl}, \mathrm{Ht}=42 \%$, erythrocytes $=4500000$ per cubic millimeter, white blood cell=5200 per cubic millimeter, platelets $=200000$ per cubic millimeter, $\mathrm{TGO}=12 \mathrm{UI} / \mathrm{l}, \mathrm{TGP}=10 \mathrm{UI} / \mathrm{I}$ TroponinT $=0,05 \mu \mathrm{g} / \mathrm{L}, \mathrm{CPKMB}=10$ $\mathrm{mcg} / \mathrm{L}$. Echocardiography was normal. In this stage, with a diagnosis of lipothymia and arterial hypotension a treatment with injectomat with dopamine with a rhythm of administration $0.2 \mathrm{ml}$ per hour was initiated, but the value of blod pressure decreased:BP=50/30 mmHg. An emergency abdominal ultrasaound was performed, which reveals an increased quantity of intraabdominal fluid, which does not allow the visualization of the internal organs. A paracentesis was performed and surprisingly - the liquid was fresh blood, so the patient had hemoperitoneum. The patient was transferred in the General Surgery Department for emergency laparotomy. The real diagnosis was: broken and bleeding right ovarian tumor with hemoperitoneum. A haemoperitoneum suction drainage was performed and a right ovarian resection, with favorable evolution and the saving of the patient's life.
\end{abstract}

Summary of the results: The onset of this clinical case was surprisingly through with a vital risk complication, the apparition of the hemoperitoneum, which clinically it manifested through lypothymia and hypotension $(\mathrm{BP}=60 / 40$ $\mathrm{mmHg}$ ).

Conclusions: Principal particularity of this clinical case report was the sudden onset with a vital risk complicationhemoperitoneum and a hemorrhagic shock tendency. The hemoperitoneum is the single situation of acute surgical abdomen, in wich the clinical signs of peritoneal irritation is possible do not appear.

Keywords: Lipothymia; Arterial hypotension; Ovarian tumor

\section{Introduction}

The main objectives of this case presentation was for me to determine the real cause of a 50 year old patient that presented into emergency service for an episode of lipothymia and arterial hypotension $(\mathrm{BP}=60 / 40 \mathrm{mmHg})$

\section{Patient and Methods}

I am presenting the clinical case of a 50 year old patient that come at the emergency service for an episode of lipothymia and arterial hypotension $(\mathrm{BP}=60 / 40 \mathrm{mmHg})$. During the medical objective examination the following are observed:pale skin (Figure 1) tachycardia (HR=105/min) (Figure 2), normal vesicular sound, value of the blood pressure was $\mathrm{BP}=60 / 40 \mathrm{mmHg}$ (Figure 3 ).

At palpating the abdomen was: insensitive, soft, elastic, participating at the respiratory movements. Negative Blumberg sign (without clinical signs of peritoneal irritation, without tenderness) (Figure 4). Normal rectal touch: (Figure 5) - normal aspect of stool - without pain during the maneuver.

The paraclinical investigations have shown: EKG sinus rithm, heart rate $=105$ bates $/$ minutes -sinustahycardia, ESR $=24 / 42 \mathrm{~mm} / 1 \mathrm{~h}$, fibrinogen $=580 \mathrm{mg} / \mathrm{dl}, \mathrm{Hb}=11 \mathrm{~g} / \mathrm{dl}, \mathrm{Ht}=42 \%$, erythrocytes $=4500000$ per cubic millimeter, white blood cell $=5200$ per cubic millimeter, plateles $=200000$ per cubic millimeter, Troponin $=0,05 \mu \mathrm{g} / \mathrm{L}, \mathrm{CPKMB}=10$ $\mathrm{mcg} / \mathrm{L}, \mathrm{TGO}=12 \mathrm{UI} / \mathrm{l}, \mathrm{TGP}=10 \mathrm{UI} / \mathrm{l}$. Echocardiography was normal and otherwise everything within normal limits. In this moment, the diagnosis was: lipothymia and arterial hypotension of unknown etiology. In this stage, with a diagnosis of lipothymia and arterial hypotension of unknown etiology, and in order to sustain the blood pressure, a treatment with injectomat with dopamine (five dopamine ampoules in $500 \mathrm{ml}$ saline solution) with a rhythm of administration $0.2 \mathrm{ml}$ per hour i.v. was initiated. After therapy intra venous continue with dopamine, the patient's evolution was with maintaining the blood pressure level at the same values for approximately 20 minutes and after that $\mathrm{BP}$ gets to a lower value of $\mathrm{BP}=50 / 30 \mathrm{mmHg}$. I am mentioning that during the anamnesis no source of bleeding has been not revealed (haematemesis, melaena, or meno - metrorrhagia - thus the patient being in menopause). In this moment, an emergency abdominal ultrasaound was performed, which reveals an increased quantity of intraabdominal fluid, which does not allow the visualization of the internal organs (Figure 6).

*Corresponding author: Manuela Stoicescu, Faculty of Medicine and Pharmacy Medical Disciplines Department, University of Oradea, Romania, E-mail: manuela_stoicescu@yahoo.com

Received December 11, 2012; Accepted January 15, 2013; Published January 21, 2013

Citation: Stoicescu M (2013) The Real Intraoperatve Diagnosis of a Patient with Lipothymia and Arterial Hypotension. J Transplant Technol Res 3: 119. doi:10.4172/2161-0991.1000119

Copyright: (C) 2013 Stoicescu M. This is an open-access article distributed unde the terms of the Creative Commons Attribution License, which permits unrestricted use, distribution, and reproduction in any medium, provided the original author and source are credited. 
Citation: Stoicescu M (2013) The Real Intraoperatve Diagnosis of a Patient with Lipothymia and Arterial Hypotension. J Transplant Technol Res 3: 119. doi:10.4172/2161-0991.1000119

Page 2 of 3

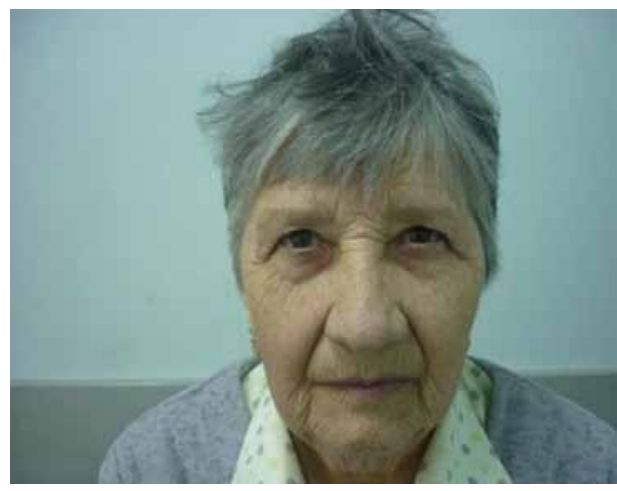

Figure 1: Pale skin.

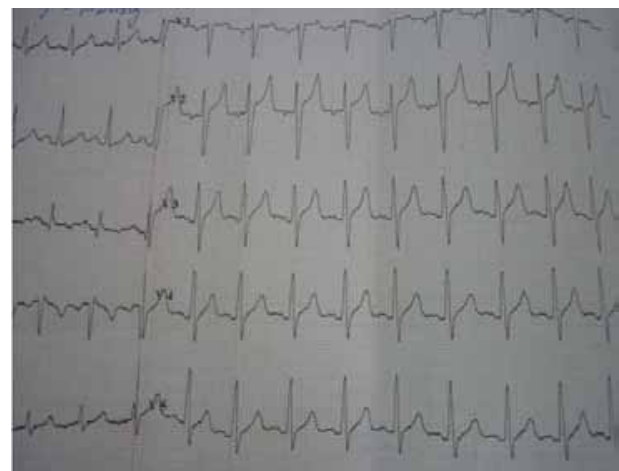

Figure 2: EKG-Sinusal tachycardia (HR=105/min).

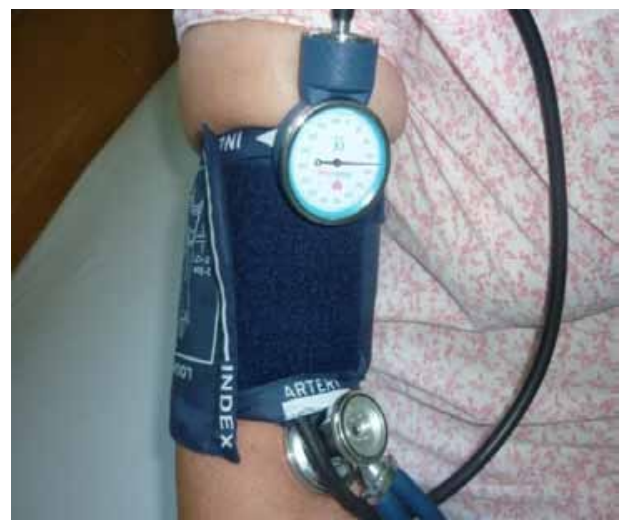

Figure 3: Blood pressure-arterial.

A question was raised wether this could be an ascitic fluid, but this was totally inconsistent with the patient's clinical aspect, because she did not presented any clinical sign of liver cirrhosis, heart failure or nephrotic syndrome, did not have edema of the legs or fluid collections in other serous, reason for which a paracentesis was performed. After conducting paracentesis, it is surprisingly that the extracted liquid consists in fresh blood, so the conclusion was that the patient had hemoperitoneum (Figure 7).

Of course, in that very instance the main problem was, what could be the hemoperitoneum etiology, since the patient not had no history of trauma. The diagnosis in that moment was:hemoperitoneum,lipothymia, arterial hypotension, tendency at a hemorrhagic shock. The level of the $\mathrm{Hb}, \mathrm{Ht}$ and red blood cell was not

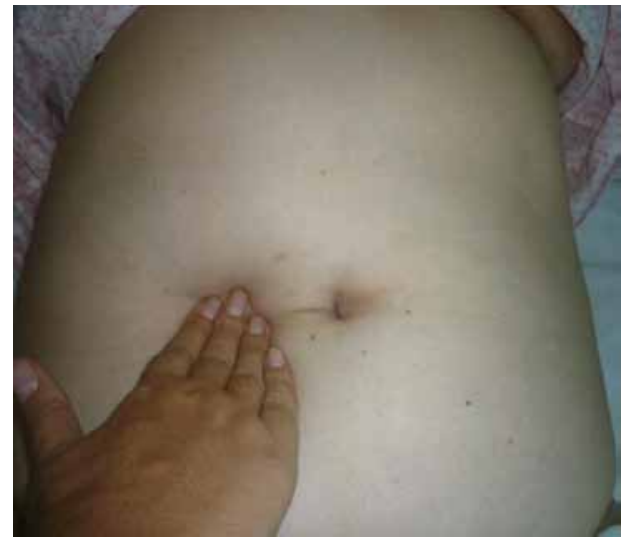

Figure 4: Palpating the abdomen Hypotension $(\mathrm{BP}=60 / 40 \mathrm{mmHg})$ insensitive soft, elastic.
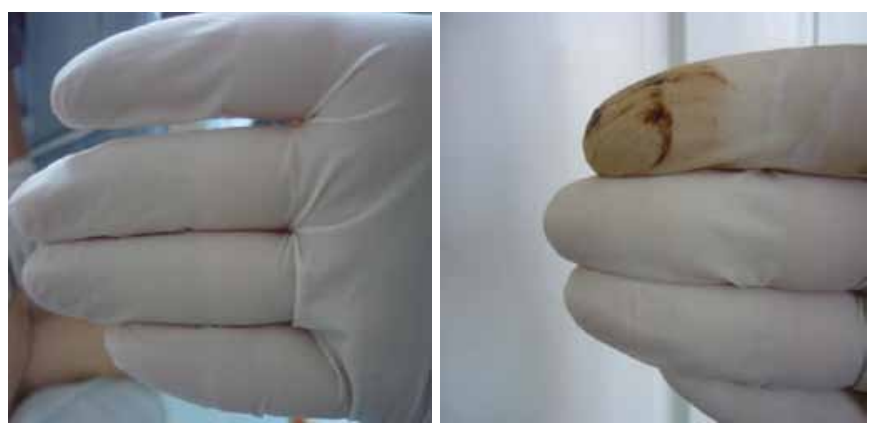

Figure 5: Rectal touch- Normal colour of stool - brown excluded melaena.

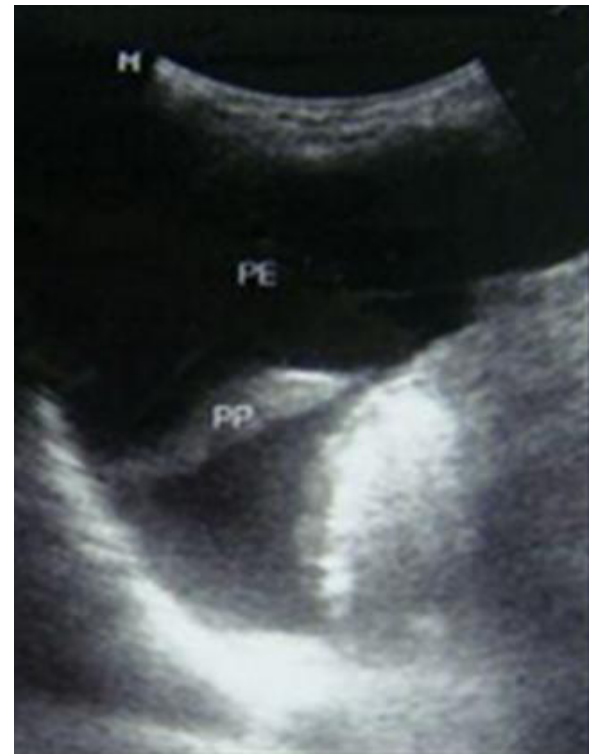

Figure 6: Abdominal ultrasound - Increased quantity of fluid to cover the internal organs. 
repeated, because the status of the patient was grave and to don't lost precious time.

\section{Results}

In that moment, the patient was transferred in the General Surgery Department for emergency laparotomy. After the surgery, it is indicated that the real diagnosis of the patient was reveals: broken and bleeding right ovarian tumor (Figure 8 ) with hemoperitoneum. A haemoperitoneum suction drainage was performed and a right ovarian resection, with a favorable outcome after surgery and the saving of the patient's life. The real diagnosis was discovered only after surgerylaparotomy. The final intraoperative diagnosis of the patient was: right ovarian tumor broken, and haemoragic with haemoperitoneum, faintness, hypotension and a tendency for hemorrhagic shock.
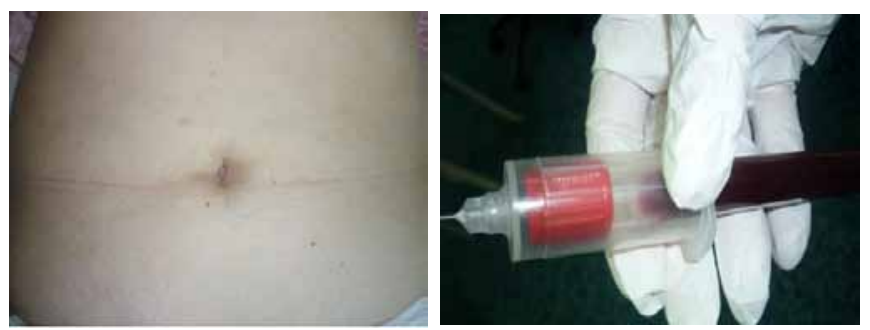

Figure 7: Paracentesis revealed fresh blood.

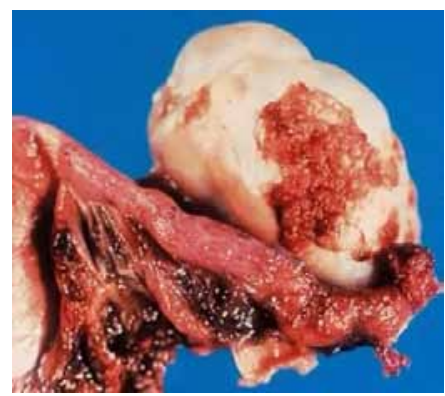

Figure 8: Right ovarian tumor broken, and bleeding post surgery.

\section{Discussions}

The onset of this clinical case was surprisingly through with a vital risk complication, the apparition of the hemoperitoneum, which clinically developed through lypothymia and hypotension $(\mathrm{BP}=60 / 40$ $\mathrm{mmHg}$ ). Although that the patient's clinical aspect could be suggested an active bleeding due to the: pale skin, sinus tachycardia, faintness, hypotension, and decreased blood pressure under dopamine tretment, it's source could not be detected from the first instance.

\section{Conclusions}

- Thesuddenonsetoftheclinicalaspectwithavitalriskcomplication / hemoperitoneum and a hemorrhagic shock tendency. -The hemoperitoneum is the single situation of acute surgical abdomen, in which the clinical signs of peritoneal irritation is possible do not appear. Referred about the abdominal pain it is possible to exist before happened the event of lipothymia and low blood pressure at home, but I don't have this information because in this conditions of bed status of the patient she can't story about this.

- Any intra-abdominal fluid that is found by performing ultrasound should be imediately punctured, in order to preclude the existence of a vital risk hemoperitoneum for not be mistake interpreted such as simple fluid of ascites.

- The lack of an important anemic syndrome, because the symptoms were at onset and the grave status of the patient do not permit to repeated this values because, to don't lost time, the patient was immediately transferred at the General Surgery Department.

- The presence of the inflammatory syndrome could have suggested the presence of a tumor.

- Clinical signs of active bleeding were present: pallor, tachycardia, hypotension, faintness, but the bleeding source could not be detected from the very begining.

- The hemoperitoneum is one of the most dangerous emergencies in the medical practice. That we don't put the correct diagnosis at the time it is possible to lose the patient's life. Every intrabdominal fluid discovered after abdominal echo need to be punctured immediately to exclude hemoperitoneum for not be confuseded such as simple fluid of ascites and to save the patient's life. 